\title{
Assessments of patients with a 5-category or a 3-category practical classification system: validaty and practicality
}

\author{
Knibbe, J.J. ${ }^{*}$, N.E.Knibbe, ${ }^{1}$, E.Waaijer ${ }^{2}$, \\ LOCOmotion, Research in Health Care, Brinkerpad 29, 6721 WJ Bennekom, The Netherlands.
}

\begin{abstract}
In order to implement preventive programs to prevent back pain in nurses, assessments of the degree of passivity and mobility of patients is imperative. After all, the load in health care ergonomics, is often the patient. The degree of cooperation or resistance determines the load on the back for the nurses and the necessity of the use of lifting devices like patient lifters or sliding sheets. These assessments must be done in both a practical and a reliable way. For this purpose a 3-category and a 5category system to assess the degree of patient mobility and passivity was developed and tested. The results are presented on the poster.
\end{abstract}

Keywords: ergonomics; prevention; back pain; patient assessment, nurse

\section{Introduction}

Occupational back pain among nurses still leads to high costs for health care facilities and personal suffering for nurses [1-4]. In order to reduce this problem a national approach was undertaken in the Netherlands by means of so-called convenants. In each health care sector agreements supported by signed commitment by all relevant parties led to the development of guidelines for practice and considerable support for the implementation process. As one of the first steps in this process, healthcare organisations in the Netherlands have developed guidelines for each patient handling activity, which stipulate a total ban of manually lifting passive patients, strict application of ergonomic devices, and specified work techniques during patient washing and wound care.

In order to implement this, the assessments of the degree of passivity and mobility of patients is a core element of the approach. This must be done in both a practical and a reliable way. For this purpose a 3category and a 5-category system to assess the degree of patient mobility and passivity was developed.

\section{Assessment of functional mobility and guidelines for practice}

The required use of specific ergonomic devices during patient handling activities is based on the assessment of the mobility of the patients. At first three mobility categories were distinguished; (1) patients who are independent or need a little bit of help due to insecurity; (2) patients who are unable to take care of themselves and need help from the nurses; and (3) patients who are completely dependent of the nurses.

For transferring a patient, a stand-up hoist is compulsory for a patient in the second category and a mobile hoist is necessary for a patient in the third category. An electric adjustable bed is required when

\footnotetext{
${ }^{*}$ Corresponding author. E-mail: j.j.knibbe@planet.nl.

${ }^{11}$ LOCOmotion, www.locomotion.nu

${ }^{2}$ Arjohuntleigh, elly.waaijer@arjohuntleigh.com
} 
a patient is being washed or dressed on the bed and an adjustable shower chair is required when a patient is showered in sitting position for a patient both in the second and third category. For repositioning patients within the bed, an electrical adjustable bed and slide sheet or overhead pole is compulsory for a patient in the second as well as the third category. A compression stocking slide (easy slide) should always be used for putting on and pulling out compression stockings of a patient, independent of the mobility category of the patient.

In the course of the implementation process the demand for a further specification of the mobility categories was apparent. Therefore a 5 category system was developed based on the ICF- 2 and ergonomic requirements. Its content was $100 \%$ correlated to the three category system that was in use initially. The differences between the two systems ( 3 and 5 categories) are mainly related to issues of quality of care. For example the need for an ergonomic device can be similar, but the need of activating the patient is specified in the 5 category system, whereas it is not in the 3 category system.

\section{Method and research question}

On the poster the results of an international validation study in four countries will be presented. The basic question was if it is possible to use these categories in a practical, reliable and valid way. And if so, what the advantages or disadvantages of the 5category system are over the 3-category system.

The study took place in four countries (UK, USA, Germany and The Netherlands). From each country 4-5 facilities participated: two from a long stay, nursing home like setting and two from (a more) acute care setting.

The results of the following three data sources ('triangulation') were combined. Each of these tools had a slightly different classification system and the results will be compared.

1. the results of the Care Thermometer (CT)

2.the results of the StaDyMeter (SDM): an activity $\log$ based on self-registration

3.the results of the RiskRadar (RR): a more general exposure assessment (not self-administered).

\section{Results}

A total of 17 facilities participated in de study and they were evenly spread among the four countries, half a more acute care setting and the other half a more residential care type setting. A total number of 301 nurses participated. The response rate ranged from $62 \%-100 \%$. The nurses registered 4919 activities on their activity logs (StaDyMeter): averaging out towards a response rate of $82 \%$. Almost the same number and same group of nurses (295) replied by filling in a RiskRadar on their activities. This pointed to an overall response rate of $76 \%$. And finally a total number of 1808 patients were assessed with the CT. This was a response rate of $96 \%$. This data was collected from the same wards as the nurses worked on.

\section{Conclusions}

The results will be ready in the autumn of 2011 and will be presented on the poster during IEA 2012.

\section{References}

[1]Hignett, S., Crumpton, E., Ruszala, S., Alexander, P., Fray, M., Fletcher, B. (2003). Evidence based patient handling: systematic review. Nursing Standard, 17 (33), 33-36.

[2] Jansen, J., Morgenstern H., Burdorf, A. (2004). Doseresponse relations between occupational exposures to physical and psychosocial factors and the risk of low back pain. Occupational Environmental Medicine, 61(12): 972-979.

[3] Knibbe, J.J., Friele, R.D. (1999). The use of logs to assess exposure to manual handling of patients, illustrated in an intervention study in home care nursing. International Journal of Industrial Ergonomics 4 (24), 445-454.

[4] Knibbe, J.J., Knibbe, N.E., Geuze, L. (2008). Een hap uit een gegroeide olifant, vierde nationale monitoring fysieke belasting, SOV\&V, Den Haag (in Dutch). Fourth National Monitoring, Unions \& Employers organizations, The Hague. 